\title{
(10h

Clinical outcome of design modifications to the CLS Spotorno Stem in total hip replacement

\author{
ANGELO GRACEFFA ${ }^{1}$, PIER FRANCESCO INDELLI ${ }^{2}$, LEONARDO LATELLA ${ }^{1}$, PAOLO POLI ${ }^{1}$, \\ ALEXANDER FULCO ${ }^{3}$, MASSIMILIANO MARCUCCI ${ }^{1}$
}

${ }^{1}$ Centro Eccellenza Sostituzioni Articolari Toscana (CESAT), Clinica Ortopedica Università di Firenze, Florence, Italy; Fondazione Onlus “...In Cammino...", Fucecchio, Italy

2 The Department of Orthopaedics and Bioengineering, Stanford University School of Medicine, Stanford, USA

${ }^{3}$ Georgetown University, Washington DC, USA

\begin{abstract}
Purpose: historically, the original CLS Spotorno Stem has demonstrated excellent survival. The design of this stem was recently modified, resulting in the introduction of a shorter, modular version (CLS Brevius). The purpose of the current study was to evaluate the functional, radiological and survivorship outcomes of the cementless CLS Brevius Stem in a multi-surgeon, single center, consecutive series study at two years post-surgery.

Methods: the Authors performed 170 total hip arthroplasties in 155 patients using the shorter, tripletaper stem design (CLS Brevius). The patients' diagnoses were primary hip osteoarthritis $(\mathrm{OA})$ in $74.4 \%$, secondary hip OA in $22.6 \%$, and post-traumatic hip $\mathrm{OA}$ in $3 \%$. All operations were performed through a mini-posterior approach, with the patient in the lateral decubitus position. The mean follow-up was 32 months (24-44 months). Outcome was assessed using the Harris Hip Score (HHS).

Results: the mean HHS improved from 32 preoperatively to 92 points at final follow-up, while the stem survival rate was $99.4 \%$.

Overall, the results were excellent in148 hips (87\%), good in 14 hips $(8.2 \%)$, fair in six hips $(3.6 \%)$, and poor in two hips $(1.2 \%)$. Intraoperative complica-
\end{abstract}

\section{Corresponding Author:}

Pier Francesco Indelli, MD, PhD

The Department of Orthopaedics and Bioengineering, Stanford University School of Medicine, Stanford, USA

3801 Miranda Avenue, 94304 Palo Alto, USA

E-mail: pindelli@stanford.edu tions included a calcar fissure in three hips $(1.7 \%)$. Correct femoral offset was reproduced in $97 \%$ while the planned center of hip rotation was achieved in $98 \%$. Only one hip underwent early stem revision; this was due to major subsidence.

Conclusions: the modified CLS stem design showed excellent short-term results with a low rate of early postoperative complications. One of the main findings of this study was the high correlation between the planned femoral offset and center of hip rotation and the final radiographic measurements. This high reproducibility, which indicates the ability of the system to restore normal hip anatomy, is indeed due to the extensive modularity that characterizes this stem system. Long-term follow-up studies are necessary to fully compare the outcomes of the new design with its highly successful predecessor.

Level of Evidence: Level IV, therapeutic cases series.

Keywords: arthroplasty, CLS, femoral stem, hip, osteoarthritis.

\section{Introduction}

Since the technique was first introduced in 1960, total hip arthroplasty (THA) has become a remarkably successful operation for treating hip osteoarthritis (OA). Over this time, the technique has been extended to younger patients, too, and cementless implants have become the standard fixation option in many countries, accounting for more than $90 \%$ of all THAs in the United States. This method of fixation offers bio- 
logical bone ingrowth with the potential for remodeling of the bone-implant interface, and it has resulted in excellent long-term clinical outcomes (1). Shorter stems, which are designed to achieve a more anatomical pattern of stress distribution permitting resection of less bone, are gaining popularity (2); from a clinical performance perspective these stems are also designed to reduce the incidence of mid-thigh pain, which occasionally occurs with the use of cementless stems in active patients (3).

The CLS Spotorno Stem (Zimmer GmbH, Winterthur, Switzerland) has been in clinical use since 1984 and has become one of the most widely used cementless total hip systems in the world: it is a triple-tapered stem intended mainly for proximal/metaphyseal fixation. Its design includes a trapezoidal cross-section and longitudinal fins on the anterior and posterior surfaces of the proximal part of the stem. The anchorage philosophy of the CLS Spotorno Stem is to preserve both the proximal bone and the fixation without attempting to fill the canal distally. Mallory et al. (4) reported excellent long-term survivorship of the tapered geometry in femoral implants. The above characteristics allow for early rotational stability and provide a large contact area with the trabecular bone, favoring early osseointegration and reinforcing axial stability (5). This stem is made of highly biocompatible titanium alloy designed to enhance stress transfer to the proximal femur and thereby prolong the life of the implant. Several studies have demonstrated the excellent clinical and radiological performance of this system $(6,7)$. The theoretical disadvantages of this femoral implant include its limited number of stemhead combinations (15 if using a metal head, 12 if using a ceramic head) for restoring the correct center of hip rotation, the need for an extensive surgical approach, and its "unfriendly" bone invasiveness.

Because of these limitations, the original CLS stem was modified, resulting in the creation, in 2011, of the CLS Brevius Stem (Zimmer GmbH, Winterthur, Switzerland). The main innovations of this system are its length (20\% shorter than the original design) (Fig. 1), its less prominent shoulder, and its modular neck (Kinectiv; Zimmer, Warsaw, USA); it allows the surgeon to reproduce 60 different centers of hip rotation while customizing the implant to different morphologies.

The characteristics retained from the original design

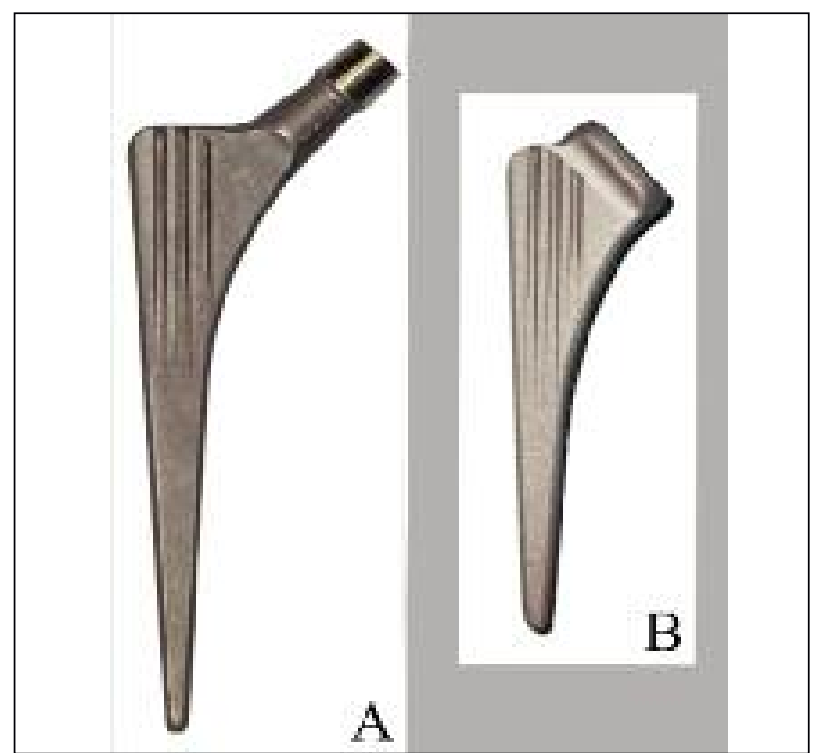

Fig. 1. The original CLS Stem (Zimmer) (A) and the modified CLS Brevius Stem (Zimmer) (B).

were the triple-taper geometry, the number of available sizes, the material (titanium), and the characteristic anterior and posterior fins (Fig. 2).

The purpose of the current study was to evaluate the functional, radiological and survivorship outcomes of the cementless CLS Brevius Stem in a multi-surgeon,

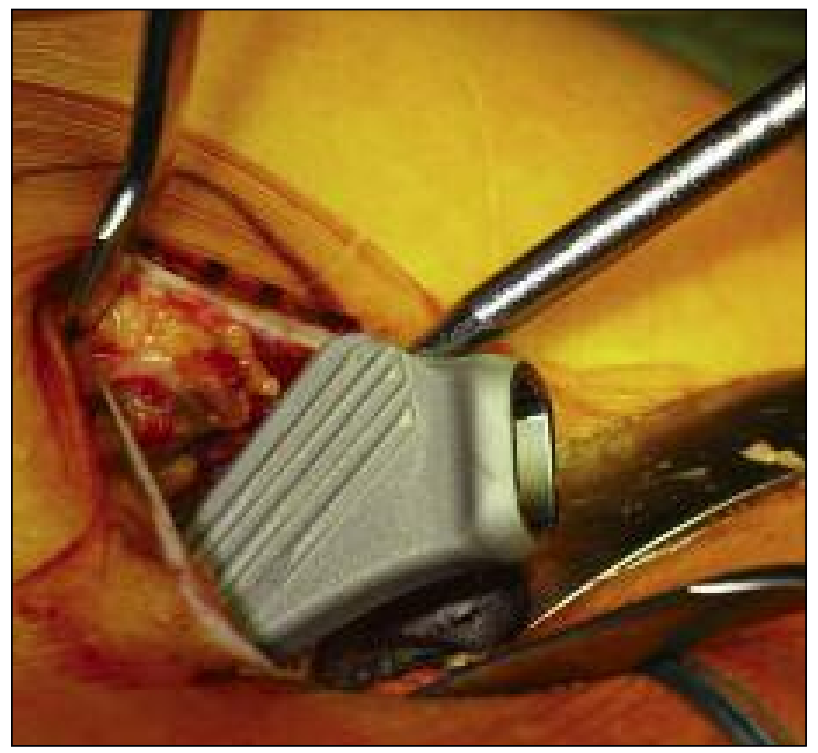

Fig. 2. Left hip. CLS Brevius Stem insertion: three longitudinal fins are present on the anterior and on the posterior surfaces of the proximal part of the stem. 
single-center, consecutive series study at two years post-surgery. The Authors hypothesized that the excellent results traditionally obtained with the CLS Spotorno Stem would not be jeopardized by any of the modifications incorporated into the CLS Brevius Stem.

\section{Methods}

This study was approved by the Institutional Review Board at our institution (Clinica Ortopedica Università degli Studi di Firenze - CESAT, Fucecchio, Italy); subsequently, informed consent was obtained from all the patients.

\section{Participants}

From April 2011 to December 2012, 170 cementless THAs were performed using CLS Brevius Stem implants in 155 patients. Fifteen of the patients underwent bilateral THA. The study group included 80 females and 75 males. The mean age of the patients at surgery was 61.8 years (range $42-67$ years). The patients had the following diagnoses: primary hip OA in $74.4 \%$, secondary hip OA in $22.6 \%$, and posttraumatic hip OA in $3 \%$. The only exclusion criterion in this consecutive series was known severe osteoporosis of the proximal femur. No patient had previously undergone surgery on the affected hip. As regards the morphology of the femoral neck, a physiological $135^{\circ}$ neck-shaft angle was observed in $45.7 \%$ of the cases, a varus neck in $35.6 \%$, and a valgus neck in $18.6 \%$.

\section{Interventions}

Preoperative templating included measurement of leg-length discrepancy, center of hip rotation and offset, as well as cup and femoral stem sizing and positioning assessment, and intraoperative pelvic tilt measurement, according to the standard preoperative protocol applied at the Authors' institution, reported in a previous study (8).

All operations were performed with the patient in the lateral decubitus position after regional anesthesia (spinal or epidural). A mini-posterior approach (with an approximately $6.5 \mathrm{~cm}$ incision) was used in all cases: this technique allowed direct measurement (with a sterile ruler) for neck-level resection relative to the lesser trochanter in accordance with the preopera- tive planning. All acetabular implants were press fitted utilizing a tilt-meter connected to the cup holder (inclination $42^{\circ}$; anteversion $20^{\circ}$ ) to allow the inclination of the implant to be adjusted for pelvic tilt (Fig. 3), as measured on intraoperative X-ray. The cup inclination was adjusted by $1^{\circ}$ for every degree of pelvic tilt as established by the intraoperative $\mathrm{X}$-ray. Other intraoperative measurements taken included the distance between the lesser trochanter and the new center of hip rotation in order to avoid leg length discrepancy.

A cementless, straight CLS Brevius Stem was implanted in all cases using a standard press-fit technique. The acetabular component was a modular press-fit cementless cup in 127 hips (74.7\%) (Continuum, Zimmer, Warsaw, USA) and a non-modular press-fit cup in the other 43 cases $(25.3 \%$ ) (Maxera, Zimmer, Warsaw, USA). All patients received a ceramic-on-ceramic bearing (Biolox, Ceramtec, Plochingen, Germany).

Antibiotic prophylaxis was performed using a first generation cephalosporin, which was administered 30 minutes before the surgical incision and for 36 hours postoperatively.

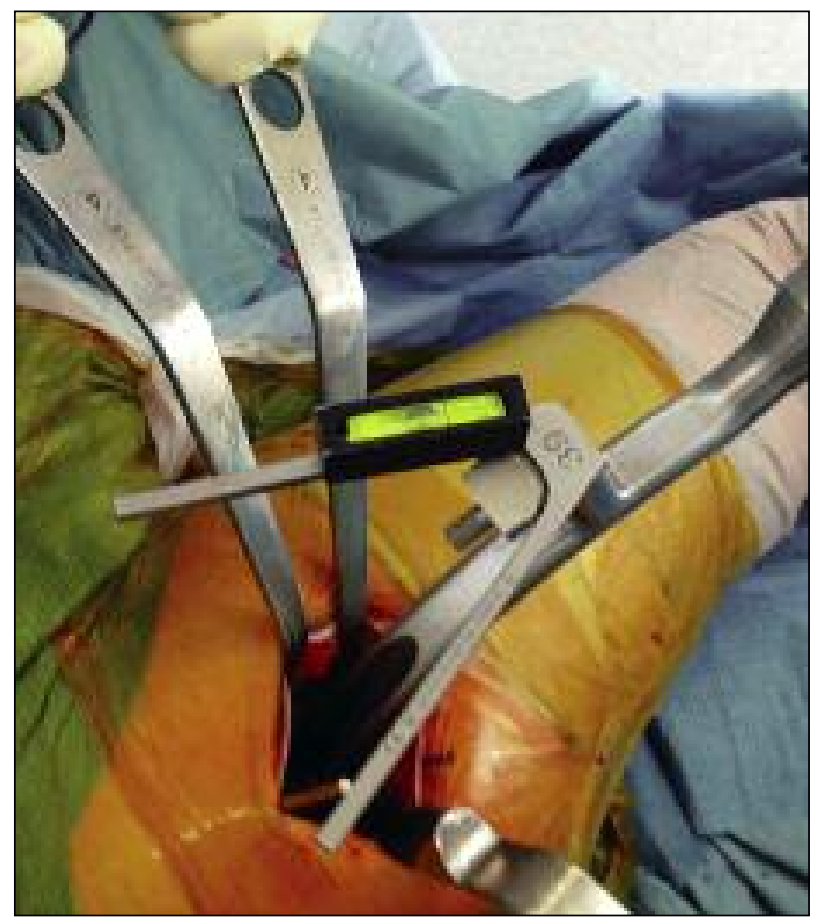

Fig. 3. Right hip. During the acetabular component positioning phase, a tilt-meter is connected to the cup holder to allow the inclination of the implant to be adjusted for pelvic tilt. 
Preoperative and postoperative color Doppler ultrasonography assessment of both lower limbs for deep venous thrombosis (DVT) was performed in all the patients, using an Esaote MyLab40 device (Genoa, Italy). Anti-thromboembolic measures included prophylaxis for DVT for 35 days postoperatively: a single daily dose of low molecular weight heparin medication (nadroparin $0.4 \mathrm{ml}$ ) was given to $32 \%$ of the patients, while a single daily dose of fondaparinux was given to $68 \%$.

All the patients used an intermittent pneumatic boot compression device for the first 24 hours postoperatively while being monitored in a sub-intensive care unit. Anti-embolism stockings were used for 30 days after surgery in all the patients.

Twelve percent of the patients received nonsteroidal anti-inflammatory drugs to prevent heterotopic ossification.

All the patients were allowed weight bearing as tolerated on the first postoperative day; they used bilateral crutches for three to five days postoperatively, and a contralateral crutch after this time. Patients abandoned walking aids only when they were pain-free and confident that they could walk on their own. While preparing the femoral canal with a series of chipped tooth broaches of increasing size, a calcar crack occurred in two of the hips; in both cases this was fixed with cerclage wiring. These two patients received a modified postoperative protocol, including avoidance of weight bearing for a period of four weeks. In these two cases, partial weight bearing with crutches was encouraged and full weight bearing (as tolerated) was suggested at 6-8 weeks after surgery.

\section{Outcome measurements}

Each patient underwent a clinical and radiological evaluation at 3 months, 6 months and 1 year after surgery, and annually thereafter. The Harris Hip Score (HHS) was used for functional assessment before surgery and at the final follow-up. All the patients were observed closely for any possible complications. The following were evaluated on standard radiographs: stem alignment, stem subsidence, endosteal bone formation, cortical hypertrophy, osteolysis, stress shielding, stem pedestal formation, heterotopic ossification according to the classification of Brooker et al. (9), and loosening of the components. In particular, as suggested by Aldinger et al. (10), stem malalign- ment was defined as a $\geq 2^{\circ}$ deviation from the longitudinal femoral axis. Stem subsidence was defined as a downward axial migration of $2 \mathrm{~mm}$ or more in relation to the greater trochanter. Radiolucent lines and areas of osteolysis were described according to the method of Gruen et al. (11) on the femoral side and according to the method of DeLee and Charnley (12) on the acetabular side. Particular attention was paid to the cup inclination in relation to the bilateral teardrop line in order to detect outliers from the desired $42^{\circ}$.

\section{Data analysis}

Statistical analysis was performed using SPSS software (SPSS 22, Chicago, Il, USA). Kaplan-Meier analysis was used to predict survival rates, taking revision for any reason as the endpoint. Pearson's chi-square test was used for analysis of the relationship between stem alignment and subsidence of the femoral stem. Results were also compared using the z-test, whether the improvement in HSS after surgery was significant or not.

\section{Results}

No patients were lost to follow-up or died during the evaluation period, with the result that all 155 patients (170 hips) were available for complete clinical and radiological analysis at the two-year follow up. The patients were monitored for a mean of 32 months (range 24-44 months). The mean HSS improved from 32 points preoperatively (range 9-58) to 92 points at two years (range 75-100); this was a highly statistically significant change with a $\mathrm{z}$-value of 27.9.

Overall, the results were excellent in 148 hips (87\%), good in 14 hips (8.2\%), fair in 6 hips (3.6\%), and poor in 2 hips $(1.2 \%)$. Post-arthroplasty trochanteric bursitis was present in 12 patients ( $7 \%$ ) at 1 year, but it persisted at the final follow-up in only 3 patients $(1.7 \%)$; all of these 3 patients had a varus modular neck.

One hundred and sixty-nine stems (99.4\%) were in situ at the time of the final follow-up. One femoral component $(0.6 \%)$ was revised 14 months after implantation because of major subsidence; in this case, the originally implanted CLS Brevius Stem, found to show neutral alignment, was substituted with another, larger CLS Brevius Stem. In one case, a Continuum acetabular component $(0.6 \%)$ was revised 
because of early aseptic loosening at 16 months postoperatively.

Kaplan-Meier analysis, used to predict survival of the CLS Brevius femoral component, was performed in all 170 hips, with revision for any reason as the endpoint. This analysis showed a survival rate of $99.4 \%$ (95\% CI, 98.3-99.8\%) at two years. The accuracy of the preoperative planning was shown by the rates of correct prediction: $98 \%$ for the acetabular component and 95\% for the femoral component. Postoperative difference in leg length ranged from $-5 \mathrm{~mm}$ to $+4 \mathrm{~mm}$ in all patients with unilateral hip OA. None of these patients had to use shoe lifts to achieve equalization of leg lengths or complained of a noticeable leg length discrepancy. The planned femoral offset was reproduced in $97 \%$, replicating within an average of $1.6 \pm 0.9 \mathrm{~mm}$ the offset on the contralateral healthy hip. The planned center of hip rotation was achieved in $98 \%$ of the hips. Femoral radiographic parameters were also evaluated. Stem alignment was neutral in 150 (Fig. 4), varus in 16 $(9.4 \%)$ and valgus in 4 hips $(2.3 \%)$. In all but one case, stem alignment at the final follow-up remained unchanged compared to what was observed on the immediate postoperative radiographs. Stem subsidence of more than $2 \mathrm{~mm}$ was observed in three femoral components (1.7\%): 2 stems showed a minor subsidence $(2-7 \mathrm{~mm})$ but were retained because the patients had no subjective symptoms and the femoral component showed no further evidence of loosening; one patient had major subsidence $(14 \mathrm{~mm})$ with loosening of the component leading to early revision. The sub-
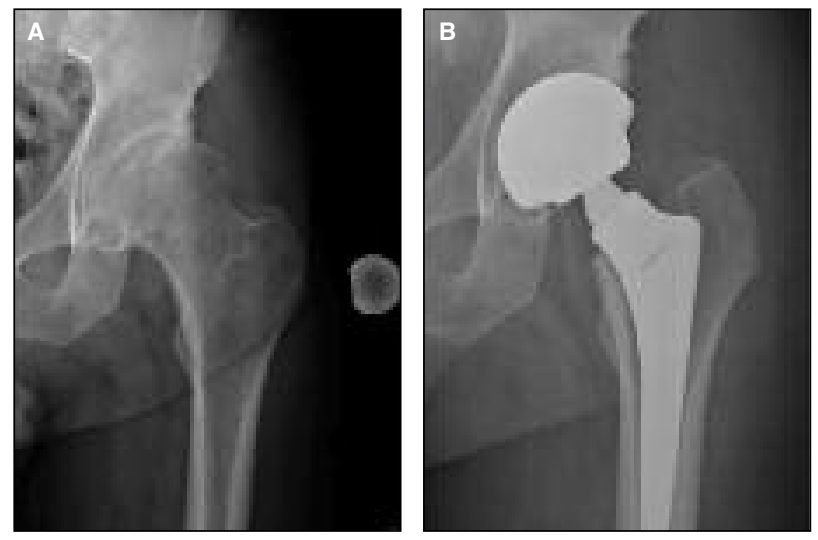

Fig. 4. Left hip. A 65-year-old man received a CLS Brevius femoral stem and a Continuum acetabular component (Zimmer): A. Preoperative radiograph. B. Two-year follow-up radiographic evaluation. sidence occurred in neutral stem alignment in two cases, and in varus stem alignment in one case. Cortical remodeling was observed in Gruen zone 2 in 4 cases $(2.3 \%)$, while a bony pedestal was found in 21 cases $(12 \%)$. Heterotopic ossification was present in 7 hips $(4 \%), 3$ in grade I and 4 in grade II. Major radiolucent lines and focal osteolysis were not found at the final follow-up.

Intraoperative complications included a calcar fissure in 3 hips $(1.7 \%)$, all treated with cerclage wiring. Perioperative complications included dislocations in 2 hips $(1.1 \%)$, which were treated with closed reduction, and thromboembolism (DVT) in 5 hips (2.9\%). There were no major perioperative complications such as superficial or deep infection, neurovascular injury or periprosthetic fracture.

\section{Discussion}

Cementless femoral components in THA have enjoyed a high success rate (13); in particular, a tapered geometry has been found to show excellent longterm survivorship with minimum radiographic evidence of stress shielding or osteolysis $(1,4)$. The original CLS Spotorno Stem showed overall survival rates of $91.5 \%$ at 23 years (14). Despite these excellent outcomes, the major limitation of the CLS Spotorno Stem was its having only 3 possible femoral-neck inclination angles $\left(145^{\circ}, 135^{\circ}\right.$ and $\left.125^{\circ}\right)$ and 5 different lengths of the metallic femoral heads $(-3.5 \mathrm{~mm} ; 0 \mathrm{~mm},+3.5 \mathrm{~mm}, 7 \mathrm{~mm}$ and $10 \mathrm{~mm})$ : multiple combinations of these parameters allowed the correct center of hip rotation to be reproduced in many, but not all, cases.

The major design modification characterizing the CLS Brevius Stem is its modularity, introduced to allow reproduction of the correct hip anatomy in the majority of cases. Other design changes compared with the original CLS Stem are its 20\% shorter length, serving to reduce bone removal, the smoothening of the stem shoulder, serving to reduce possible damage to the great trochanter in the stem insertion phase, and its smoother stem tip. The major theoretical advantage of this kind of modular femoral neck is to allow stem length adjustment without offset variation, and vice versa: at the same time, it makes it possible to select an anteverted or retroverted femoral neck 
irrespective of the stem alignment. The proximal third of the modular neck adapter is designed with a narrower diameter in order, theoretically, to improve the hip ROM.

However, the addition of a further interface (the modular femoral neck), might potentially be associated with complications like stress fractures caused by corrosion (15-17) or stem-neck disassociation, in particular during dislocation reduction maneuvers (18). Another major concern is the correlation between increased modularity and corrosion associated with titanium ion release under in vivo conditions (19), leading to local tissue infiltration and adverse periprosthetic tissue reactions (20).

Corrosion at the head-neck taper interface may be caused by three major mechanisms: crevice, fretting and galvanic. The presence of a small gap between the two components allows biological fluid to enter and create crevice corrosion at the Morse taper. Fretting corrosion can be caused by micro-movements of the head relative to the neck, the neck to the body, or the proximal body to the distal stem. Galvanic corrosion usually occurs in any mixed-metal system in which fluid is present, but it is most likely to occur in titanium alloy stems mated to CoCr heads. The Authors of the current study addressed these issues by adopting a multimodal approach: first, we always performed adequate cleaning of the trunnion before final neck impaction in order to avoid fluid interposition at the neck-body and neck-head junctions; second, we used only ceramic heads, since fretting corrosion at the head-neck junction has been shown to be significantly lower with a zirconia head than with a cobalt alloy head (21), while galvanic corrosion does not occur with ceramic heads because there is no mixed-metal system; third, we hypothesized that restoration of the femoral offset, center of hip rotation and leg length, made as anatomical as possible by the use of a modular femoral neck, might lead to inferior stresses at the neck-body and neckhead junctions and less release of corrosion products. As pointed out by van Oldenrijk et al. (22), another theoretical issue related to this new femoral implant design concerns its length. Short-stemmed implants were introduced with the aim of preserving proximal bone stock for future revisions by preventing stress shielding through metaphyseal bone loading (22). A systematic review of short stem THA reports from worldwide registry datasets showed a mean revision rate of 1.29 per 100 observed component years after primary THA, which corresponds to a revision rate of $12.9 \%$ after 10 years (23). More encouraging results were those of Rometsch et al. (24) and van Oldenrijk et al. (22), who reported a projected survivorship at 10 years of 3.8 and $7 \%$ respectively. The major limitation of these studies was the inclusion of many different stem designs, like collum, partial collum and trochanter-sparing stems.

The present study is limited by its retrospective cohort design and short follow-up: these initial results with the new, modified CLS stem are satisfactory, but certainly preliminary, and it was difficult to interpret the results statistically. Furthermore, the survival rates could have been affected by the use of two acetabular components; on the other hand, all the patients received a ceramic-on-ceramic bearing. A calcar fissure occurred in 3 hips intraoperatively, complication rate similar to that reported with the standard CLS design (25).

Stem alignment was neutral in $88 \%$, differing from previous reported results on the CLS stem. Han et al. (26), in their evaluation of a series of 95 hips treated with CLS stem, reported neutral alignment in only $60 \%$ of the cases: the difference with the current study is probably due to the fact that the CLS Brevius Stem features a less prominent shoulder, which makes it easier to achieve correct femoral alignment, avoiding varus in particular. Some Authors have reported that varus alignment of a femoral stem does not compromise the outcome when the stem has a tapered wedge shape (27): varus alignment might not be tolerated by other designs. In this study, no clinical or radiographic differences were found between varus, valgus or neutral stem alignment.

Our radiographic analysis at final follow-up showed stem subsidence of more than $2 \mathrm{~mm}$ in 3 femoral components $(1.7 \%)$, in spite of our protocol of immediate postoperative weight bearing as tolerated: one early revision surgery was required because the subsidence of the stem was considered major (14 $\mathrm{mm}$ ). The other 2 patients were asymptomatic and both had a high HHS. Historically, it has been seen that the classic CLS stem can subside minimally in the canal, subsequently achieving secondary stability through definitive press-fit as viscoelastic relaxation occurs, followed by bony ingrowth (5). Like its CLS 
predecessor, the CLS Brevius seemed to achieve secondary stability, possibly thanks to the maintenance of its geometric characteristics. One patient had an isolated revision of the acetabular component: the CLS Brevius femoral component was left in place.

While thigh pain was not found to be a postoperative issue, 12 of our patients $(7 \%)$ reported a severe trochanteric bursitis at the one-year follow-up examination; in only 3 of these this was found to persist at final follow-up. The Authors considered this complication related to the use of a varus modular neck in all 3 patients. The presence of cortical remodeling, reactive lines, pedestal formation, major radiolucent lines, or focal osteolysis was not statistically significant.

The results of Kaplan-Meier analysis with revision for stem loosening as the endpoint revealed a $99.4 \%$ survival rate of the femoral component at the twoyear follow-up.

The main finding of this study, in the Authors' opinion, was the high correlation observed between the planned femoral offset and center of hip rotation and the final radiographic measurement: 97 and 98\% respectively. This high reproducibility, which indicates the ability of the system to restore the desired hip anatomy, is indeed due to the extensive modularity that characterizes this stem system.

On the basis of the short-term outcomes obtained in this study, we conclude that the clinical and radiological results of tapered CLS Brevius stems are encouraging, especially in terms of a low rate of early complications. Preoperative planning and intraoperative assessment of the distance between the lesser trochanter and the new center of hip rotation in order to avoid leg length discrepancy would seem helpful, making it possible to insert adequately sized systems. Several reasons for our satisfactory outcomes, with few complications, must be considered. First, the concept of proximal fit with a distal taper, which characterizes the original CLS design, is retained: this is a major factor in reducing the incidence of thigh pain in our patients. The flexibility of the titanium stem is maintained too, providing for gradual load transmission and accounting for the low incidence of proximal stress shielding and distal cortical hypertrophy. However, long-term follow-up results are essential because later complications related to aseptic osteolysis, subsidence, stress shielding, and thigh pain may affect the survival of CLS Brevius Stem.

\section{References}

1. Aldinger PR, Jung AW, Pritsch M, et al. Uncemented gritblasted straight tapered titanium stems in patients younger than fifty-five years of age: fifteen to twenty-year results. J Bone Joint Surg Am. 2009;91:1432-1439.

2. Gustke K. Short stems for total hip arthroplasty: initial experience with the Fitmore stem. J Bone Joint Surg Br. 2012; 94 (11 Suppl A):47-51.

3. Renkawitz T, Santori FS, Grifka J, et al. A new short uncemented, proximally fixed anatomic femoral implant with a prominent lateral flare: design rationals and study design of an international clinical trial. BMC Musculoskelet Disord. 2008;9:147-153.

4. Mallory TH, Lombardi AV Jr, Leith JR, et al. Why a taper? J Bone Joint Surg Am 2002;84-A:81.

5. Spotorno L, Romagnoli S, Ivaldo N, et al. The CLS system. Theoretical concept and results. Acta Orthop Belg. 1993; 59:144-148.

6. Muller LA, Wenger N, Schramm M, et al. Seventeen-year survival of the cementless CLS Spotorno stem. Arch Orthop Trauma Surg. 2010;130:269-275.

7. Marcucci M, Indelli PF, Latella L, et al. A multimodal approach in total hip arthroplasty preoperative templating. Skeletal Radiol. 2013;42 (9):1287-1294.

8. Harris WH. Traumatic arthritis of the hip after dislocation and acetabular fractures: treatment by mold arthroplasty. An end-result study using a new method of result evaluation. J Bone Joint Surg Am. 1969;51:737-755.

9. Brooker AF, Bowermen JW, Robinson RA, et al. Ectopic ossification following total hip replacement. Incidence and a method of classification. J Bone Joint Surg Am. 1973;55: 1629-1632.

10. Aldinger PR, Breusch SJ, Lukoschek M, et al. A 10 - to 15year follow up of the cementless Spotorno stem. J Bone Joint Surg Br. 2003;85:209-214.

11. Gruen TA, McNeice GM, Amstutz HC. "Modes of failure" of cemented stem-type femoral components: a radiographic analysis of loosening. Clin Orthop Relat Res. 1979;(141):17-27.

12. DeLee JG, Charnley J. Radiological demarcation of cemented sockets in total hip replacement. Clin Orthop Relat Res. 1976; (121):20-32.

13. Mai KT, Verioti CA, Casey $\mathrm{K}$, et al. Cementless femoral fixation in total hip arthroplasty. Am J Orthop (Belle Mead NJ). 2010;39:126-130.

14. Evola FR, Evola G, Graceffa A, et al. Performance of the CLS Spotorno uncemented stem in the third decade after implantation. Bone Joint J. 2014;96-B:455-461.

15. Dangles CJ, Altstetter CJ. Failure of the modular neck in total hip arthroplasty. J Arthroplasty. 2010;25:1169.e5-7.

16. Ellman MB, Levine BR. Fracture of the modular femoral neck component in total hip arthroplasty. J Arthroplasty. 2013;28:196.e1-5.

17. Skendzel JG, Blaha JD, Urquhart AG. Total hip arthroplasty modular neck failure. J Arthroplasty. 2011;26:338.e1-4.

18. Fanuele J, Bernini P. Dissociation of the modular femoral stem from the metaphyseal sleeve during reduction of a total hip arthroplasty dislocation. J Arthroplasty. 2007;22:140-142.

19. Kretzer JP, Jakubowitz E, Krachler M, et al. Metal release and corrosion effects of modular neck total hip arthroplasty. Int Orthop. 2009;33:1531-1536.

20. Jacobs JJ, Urban RM, Gilbert JL, et al. Local and distant products from modularity. Clin Orthop Relat Res. 1995;(319): 94-105. 
21. Hallab NJ, Messina C, Skipor A, et al. Differences in the fretting corrosion of metal-metal and ceramic-metal modular junctions of total hip replacements. J Orthop Res. 2004; 22(2):250-259.

22. van Oldenrijk J, Molleman J, Klaver M, et al. Revision rate after short-stem total hip arthroplasty: a systematic review of 49 studies. Acta Orthop. 2014;85:250-258.

23. Labek G, Frischhut S, Schlichtherle R, et al. Outcome of the cementless Taperloc stem: a comprehensive literature review including arthroplasty register data. Acta Orthop. 2011;82: 143-148.

24. Rometsch E, Bos PK, Koes BW. Survival of short hip stems with a "modern", trochanter-sparing design-a systematic literature review. Hip Int. 2012;22:344-354.

25. Hwang KT, Kim YH, Kim YS, et al. Total hip arthroplasty using cementless grit-blasted femoral component: a minimum 10-year follow-up study. J Arthroplasty. 2012;27:15541561.

26. Han SI, Lee JH, Kim JW, et al. Long-term durability of the CLS femoral prosthesis in patients with osteonecrosis of the femoral head. J Arthroplasty. 2013;28:828-831.

27. Khalily C, Lester DK. Results of a tapered cementless femoral stem implanted in varus. J Arthroplasty. 2002;17:463-466. 